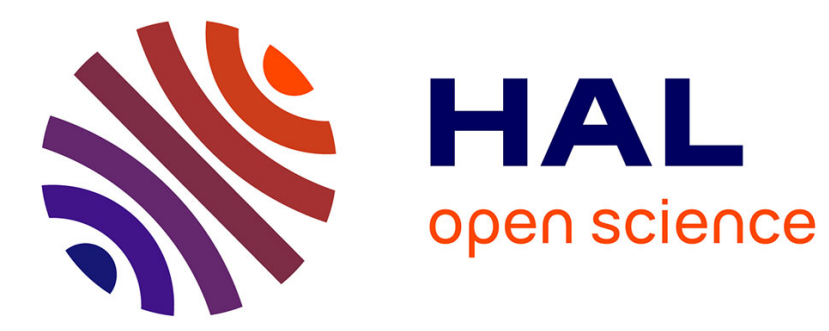

\title{
Determination of dispersion relations in quasi-stationary plasma turbulence using dual satellite data
}

Thierry Dudok de Wit, V. Krasnosel'Skikh, S. Bale, M. Dunlop, H. Lühr, S. Schwartz, L. Woolliscroft

\section{- To cite this version:}

Thierry Dudok de Wit, V. Krasnosel'Skikh, S. Bale, M. Dunlop, H. Lühr, et al.. Determination of dispersion relations in quasi-stationary plasma turbulence using dual satellite data. Geophysical Research Letters, 1995, 22 (19), pp.2653-2656. 10.1029/95GL02543 . insu-03039729

HAL Id: insu-03039729

https://hal-insu.archives-ouvertes.fr/insu-03039729

Submitted on 4 Dec 2020

HAL is a multi-disciplinary open access archive for the deposit and dissemination of scientific research documents, whether they are published or not. The documents may come from teaching and research institutions in France or abroad, or from public or private research centers.
L'archive ouverte pluridisciplinaire HAL, est destinée au dépôt et à la diffusion de documents scientifiques de niveau recherche, publiés ou non, émanant des établissements d'enseignement et de recherche français ou étrangers, des laboratoires publics ou privés. 


\title{
Determination of dispersion relations in quasi-stationary plasma turbulence using dual satellite data
}

\author{
T. Dudok de Wit, ${ }^{1,2}$ V. V. Krasnosel'skikh, ${ }^{1}$ S. D. Bale, ${ }^{3}$ M. W. Dunlop, ${ }^{4}$ H. Lühr, ${ }^{5}$ \\ S. J. Schwartz, ${ }^{3}$ and L. J. C. Woolliscroft ${ }^{6}$
}

\begin{abstract}
The joint frequency-wavenumber spectrum is one of the basic quantities for analyzing plasma turbulence. It is shown how the full spectrum can be recovered from wavefields measured by two or more satellites via spectral methods based on wavelet transforms. Compared to standard cross-correlation techniques, different branches in the dispersion relation can be resolved and quasi-stationary wavefields can be accessed. Using this new approach, low frequency magnetic field data from the AMPTE-UKS and AMPTE-IRM spacecraft are investigated and the impact of nonlinear processes on wave propagation at the Earth's foreshock is revealed.
\end{abstract}

\section{Introduction}

Two fundamental problems in the statistical analysis of plasma turbulence are the identification of deterministic dispersion relations and the separation between spatial and temporal dynamics. Both require knowledge of the joint frequencywavenumber spectrum [Lefeuvre et al., 1992] for which measurements with both temporal and spatial resolution are necessary. Although the former is rarely a problem in practice, the spatial resolution is constrained by the number of simultaneous measurements. This problem is particularly acute in space plasmas where each individual measurement requires a satellite.

In this paper, we present a technique for inferring wavefield dispersion relations using two or more satellites. While traditional approaches based on cross-spectral estimates have been successfully applied to ISEE data [Hoppe et al., 1980], it is shown how a small modification can provide new insight into wavefield properties and nonlinear processes in plasma turbulence.

\section{Turbulence at the Earth's Bow Shock}

As a typical example of strong plasma turbulence we consider magnetic field data recorded by the AMPTE-UKS and AMPTEIRM spacecraft upstream the Earth's quasi-parallel bow shock on

1 Laboratoire de Physique et Chimie de l'Environnement, CNRS, Orléans, France.

2 Now at the Centre de Physique Theorique, CNRS-Universite de Provence, Marseille, France.

3 Astronomy Unit, Queen Mary and Westfield Coll., London, UK.

4 Space and Atmospheric Physics, Imperial Coll., London, UK.

5 Inst. für Geophysik und Meteorologie, Technische Universität Braunschweig, Braunschweig, Germany.

6 Dept. of Automatic Control and Systems Engineering, University of Sheffield, Sheffield, UK.

Copyright 1995 by the American Geophysical Union.

Paper number 95GL02543

0094-8534/95/95GL-02543\$03.00 day 304 of 1984. This data set has already been discussed in [Schwartz et al., 1992; Mann et al., 1994]. The turbulence in the foreshock region reveals a great variety of wave phenomena, among which solitary structures termed Short Large Amplitude Magnetic Structures (SLAMS) [Schwartz et al., 1992] have received much attention. The SLAMS, which are a subset of more general pulsations [Thomson et al., 1990] and which are related to kinetic magnetosonic waves [Omidi et al., 1990] and to soliton Alfvén waves [Hada et al., 1989], try to propagate against the solar wind but are convected back towards the Earth. They are generally preceded at their upstream edge by high frequency whistler wavetrains. These nonlinear structures are expected to play a significant role in the dynamics of the quasiparallel bow shock, hence the large interest devoted to them.

Figure 1 shows an excerpt of the magnetic field data, which have been collected on an outbound portion of the orbit with a spacecraft separation of $|\vec{d}|=148 \mathrm{~km}$. The eigenvalues of the variance tensor [Sönnerup et al., 1967] are respectively $1,0.77$ and 0.12 , indicating that the wavefield structure is on average guite planar. The solar wind flow, the spacecraft separation vector $\vec{d}$ and the average wave-vector are all approximately parallel within $10^{\circ}$. Although this condition is not necessary for our analysis procedure, it will nevertheless ease the interpretation.

\section{Estimation of the Joint Frequency-Wavenumber Spectrum}

We start by considering the traditional procedure for estimating dispersion relations, and then show how it can be improved. Let $B(\vec{x}, t)$ be a stationary and homogeneous quantity whose ensemble-average satisfies $\langle B(\vec{x}, t)\rangle=0$. In our case, it is the projection of the fluctuating magnetic field along the direction of maximum variance. The basic assumption is that $B(\vec{x}, t)$ can be decomposed into a superposition of plane waves

$$
B(\vec{x}, t)=\iint B(\vec{\kappa}, \omega) \exp (-j(\omega t-\vec{\kappa} \cdot \vec{x})) d \vec{\kappa} d \omega+c . c .
$$

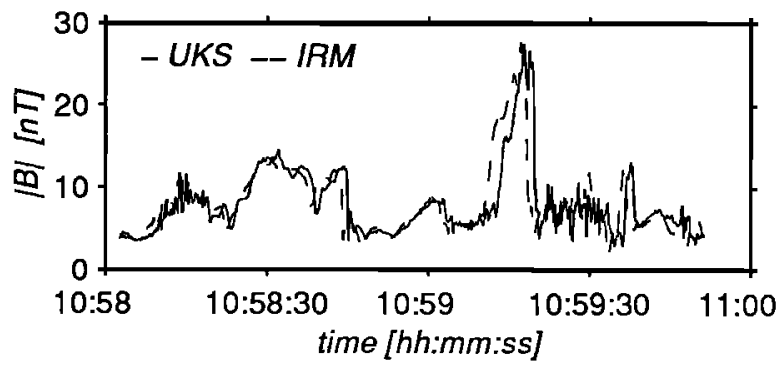

Figure 1. Time evolution of the magnetic field (excerpt), taken from the time interval 10:54:15 to 11:03:00 of day 304 of 1984. The UKS satellite is ahead (i.e. farther away from the shock front) of the IRM satellite. A typical SLAMS is observed at 10:59:20. 
where the wavefield dispersion relation reads $\vec{k}=\vec{k}(\omega)$. The quantity of interest is the joint frequency-wavenumber spectrum

$$
\mathbf{S}(\vec{\kappa}, \omega)=\left\langle B^{*}(\vec{\kappa}, \omega) B(\vec{\kappa}, \omega)\right\rangle
$$

which provides direct insight into the wavefield properties. The main difficulty arises from its estimation with a limited spatial resolution [Carlson, 1991]. With two probes, one only has access to $k_{d}=\vec{k} \cdot \vec{d} /|\vec{d}|$, the projection of the wave-vector on the separation vector. The usual approach consists in computing the cross-spectrum between two measurements and relating the crossphase to $\kappa_{d}$ by

$$
P(\vec{d}, \omega)=\left\langle B^{*}(\vec{x}, \omega) B(\vec{x}+\vec{d}, \omega)\right\rangle=|P| \exp \left(j \kappa_{d}|\vec{d}|\right)
$$

One can then in principle estimate $\vec{K}$ from the knowledge of $\kappa_{d}$ and the minimum variance orientation. This simple approach is not suited for dispersion relations which have several branches. Indeed, one can show that the outcome of Eq. 3 is not the wavenumber itself but its first moment $\overline{\mathbf{K}}_{\mathbf{d}}$ :

$$
\bar{\kappa}_{d}(\omega)=\int \kappa_{d} S(\vec{\kappa}, \omega) d \vec{\kappa} / \int S(\vec{\kappa}, \omega) d \vec{\kappa}
$$

This problem, however, can be overcome by first estimating $\boldsymbol{\kappa}_{\mathrm{d}}$ for each ensemble in Eq. 3 and subsequently building its distribution function vs $\omega$ [Beall et al., 1982]. This yields the socalled local spectrum

$$
S(\kappa, \omega)=<\frac{1}{2}\left(B^{*}(\vec{x}, \omega) B(\vec{x}, \omega)+B^{*}(\vec{x}+\vec{d}, \omega) B(\vec{x}+\vec{d}, \omega)\right) \delta\left(\kappa_{d}-\kappa\right)>
$$

where $K_{d}$ is calculated for each ensemble using Eq. 3. This local spectrum converges towards the true one $\left(\mathrm{Eq}_{\overrightarrow{.}}\right.$ 2) provided that the condition of planarity is satisfied and that $\overrightarrow{\boldsymbol{k}} \cdot \vec{d}<2 \pi$. In the case of sampled data, one just has to consider the discrete equivalent of $S(\kappa, \omega)$, which is a histogram vs $\kappa$ and $\omega$ with discrete cells.

With this improvement, we can now resolve different branches in the dispersion relation. The applicability of this technique, however, is often limited in practice by the conditions of planarity, stationarity and homogeneity, which are too restrictive. We therefore loosen these constraints and let the wavefield be composed of plane wave packets rather than plane waves. From a practical point of view, this means replacing Fourier time transforms by wavelet transforms [Farge, 1992]. The wavelet transform of $B(\vec{x}, t)$ is

$$
B(\vec{x}, a, \tau)=\int B(\vec{x}, t) \frac{1}{\sqrt{a}} h^{*}\left(\frac{t-\tau}{a}\right) d t
$$

where $h(t)$ is the analyzing wavelet, $t$ its position and a its scale. In this paper, Morlet wavelets

$$
h(t)=\frac{1}{\pi^{1 / 4}} \exp (-2 \pi j t) \exp \left(-t^{2} / 2\right)
$$

are used because of their good simultaneous time-frequency resolution. Their scale $a$ is related to the instantaneous angular frequency by $\omega=2 \pi / a$. The estimation of $S(\kappa, \omega)$ is again carried out using Eq. 5 , but the ensemble average is now replaced by an average over $t$. In practice, both high time resolution (to improve the statistics of the histograms) and good frequency resolution are needed. Wavelets are appropriate for such purposes, because their time resolution is adapted to the frequency band of interest [Meyer et al., 1993]. The wavelet transform not only considerably improves the statistical robustness of the spectral estimates but it also allows transient and soliton-like features to be better resolved. Thus, it is a natural tool for analyzing quasistationary turbulence.

\section{The Dispersion Relation and Nonlinear Effects}

The joint frequency-wavenumber spectrum estimated from the AMPTE data is shown in Figure $2 a$. We recall that the abscissa represents $k_{d}=\vec{k} \cdot \vec{d} /|\vec{d}|$. Since, however, $\vec{k}$ and $\vec{d}$ are in this instance on average colinear, the abscissa is almost equivalent to the magnitude of the wave-vector. Note also that all quantities are expressed in the satellite frame, in which the turbulence is convected by the solar wind flow. This effect will be discussed further below. For visualization purposes, we have artificially unfolded the spectra by extending the wavenumber domain to twice the Nyquist limit. With this, ridges can be followed without discontinuities throughout the $(k, f)$ plane (where $k=k / 2 \pi$ and $f=\omega / 2 \pi)$.

The spectral density depicted in Fig. $2 \mathrm{a}$ is by definition heavily weighted by large amplitude fluctuations. One may, however, also consider adapting the weighting to the fluctuation level. This opens the interesting perspective of investigating amplitudedependent properties of the turbulence. A particular choice consists in putting equal weights on all the fluctuations, regardless of their amplitude. We do this by setting $B(\vec{x}, \omega) \equiv 1$ in Eq. 5 . The result, shown in Figure $2 b$, is simply a probability density as a function of $\kappa$ for each $\omega$.

A comparative study of Figures $2 a$ and $2 b$ now allows us to assess the impact of nonlinear processes on turbulence. Indeed, these figures respectively reveal the dynamics of the large and the small amplitude fluctuations. A first result is the occurrence of a unique and linear dispersion branch in Fig. $2 b$, and consequently the absence of the dispersive behavior one would expect from finite Larmor radius and Hall term effects. Some such dispersive effects, however, may be hidden by the low signal-to-noise ratio of Fig. 2b, in which much weight is given to small fluctuations and noise. Figure $2 a$, in contrast, clearly reveals two branches which coincide at low frequencies. These two branches most probably correspond to the fast magnetosonic (I) and to the Alfven (II) mode. Such modes are known to overlap at low frequency in the quasi-parallel case but their unambiguous identification would require a simultaneous analysis of the fluctuating electron density.

Given these results, we may attribute the observed bifurcation to the role played by nonlinear effects, whose main manifestation is an amplitude-dependent frequency shift [Whitham, 1973]. A comparison between Figures $2 \mathrm{a}$ and $2 \mathrm{~b}$ in this sense clearly illustrates the impact of nonlinear processes on wave propagation. It also strongly recalls the behavior of nonlinear waves which fall in the framework of the derivative nonlinear Schrödinger equation [Mjølhus et al., 1988]. Indeed, this equation also gives rise to an amplitude-dependent dispersion relation $\omega=v_{A} K \pm \alpha \kappa^{2}+\beta \kappa A^{2}$ where in the cold plasma approximation $\alpha=v_{A} / 4 B_{0}^{2}$, $\beta=v_{A}^{2} / 2 \Omega_{i}, \quad B_{0}$ is the average magnetic field, $\Omega_{i}$ the ion cyclotron frequency, $v_{A}$ the Alfven velocity and $A$ the wave amplitude.

We finally note that branch II has a nonlinear dispersion, which means that the wave group and phase velocities differ. The competition between this effect and the nonlinear steepening of the SLAMS has been shown [Dudok de Wit et al., 1995] to be one of the causes for the occurrence of whistler waves at the leading edge of SLAMS. A comprehensive analysis of these results is presently the subject of a forthcoming publication. 
(a) Spectrum

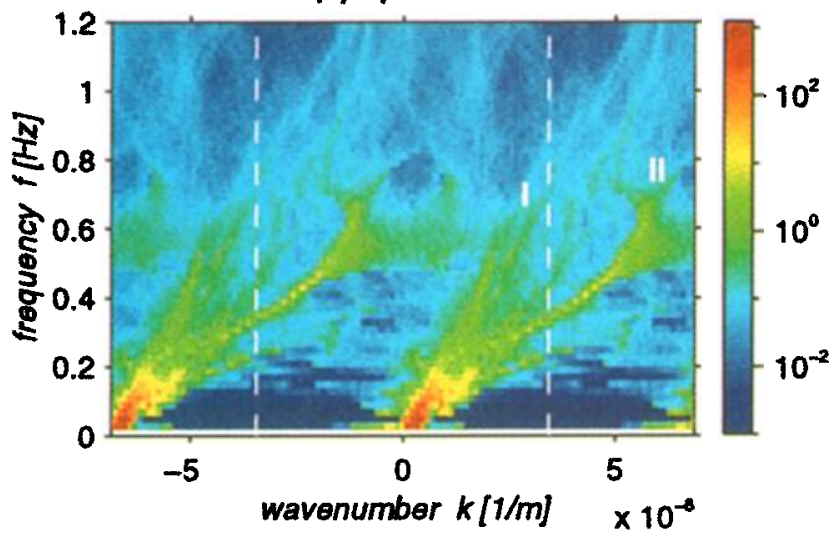

(b) Distribution function

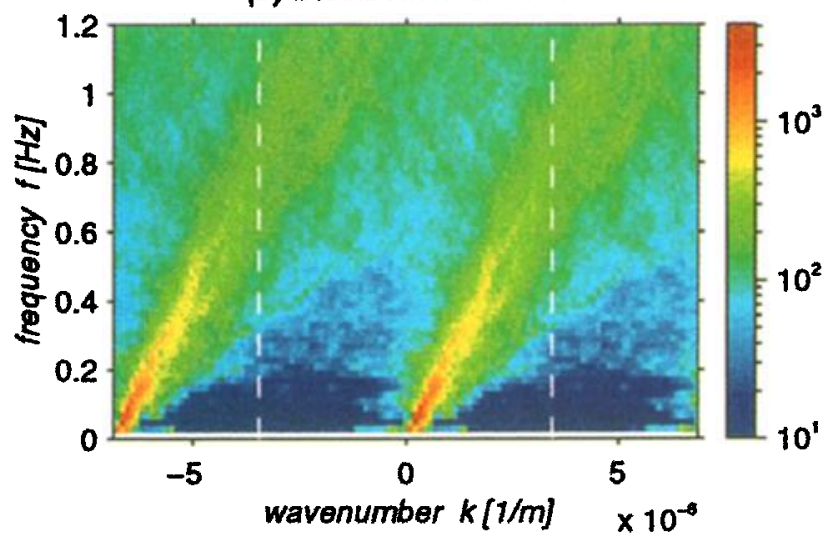

Figure 2. Representation of the joint frequency-wavenumber spectrum (a) and the probability density (b) in the spacecraft frame. Positive wavenumbers correspond to an earthward propagation. The dashed rectangle represents the principal domain limited by the Nyquist wavenumber, which has been artificially unfolded. The ridge originating from $\left(k=-6 \cdot 10^{-6}, f=0\right)$ is an artefact of this unfolding. The number of samples is 4120 and the sampling rate $8 \mathrm{~Hz}$.

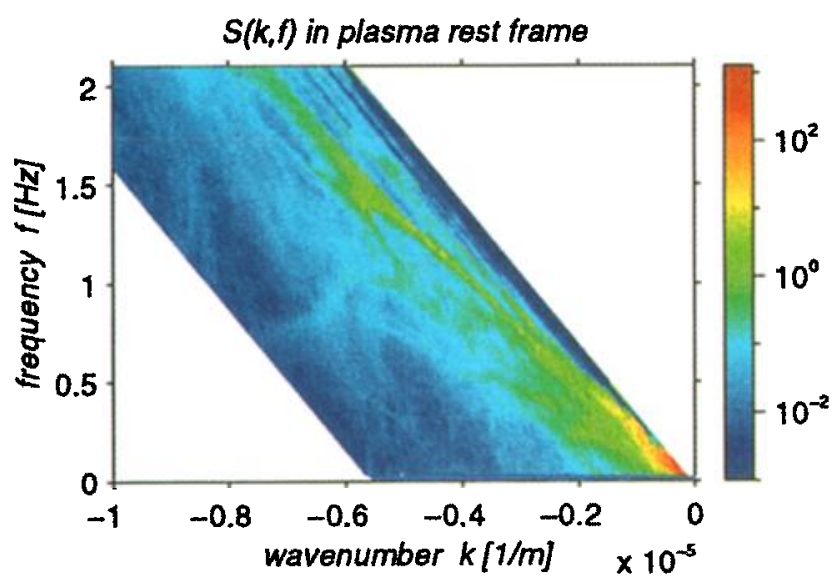

Figure 3. The wavefield spectrum after correction of the Doppler shift induced by the solar wind flow. The waves now propagate sunwards.

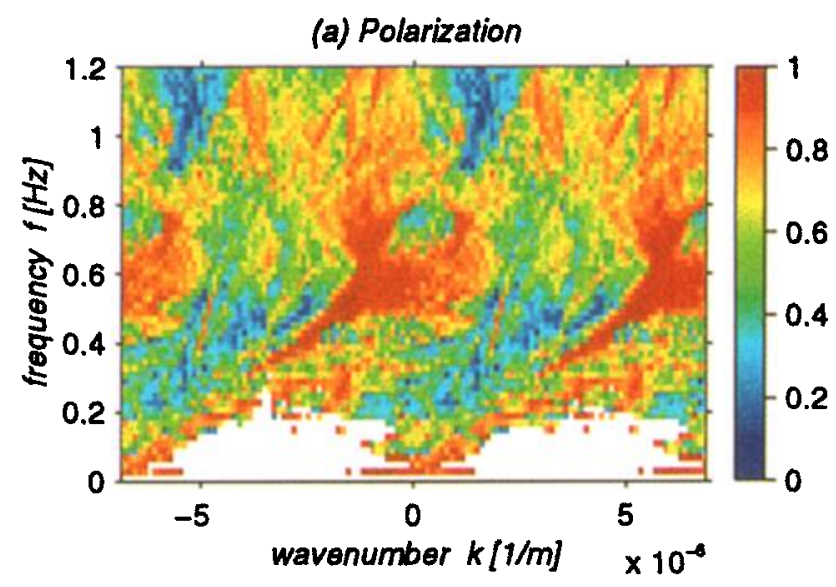

(b) Ellipticity

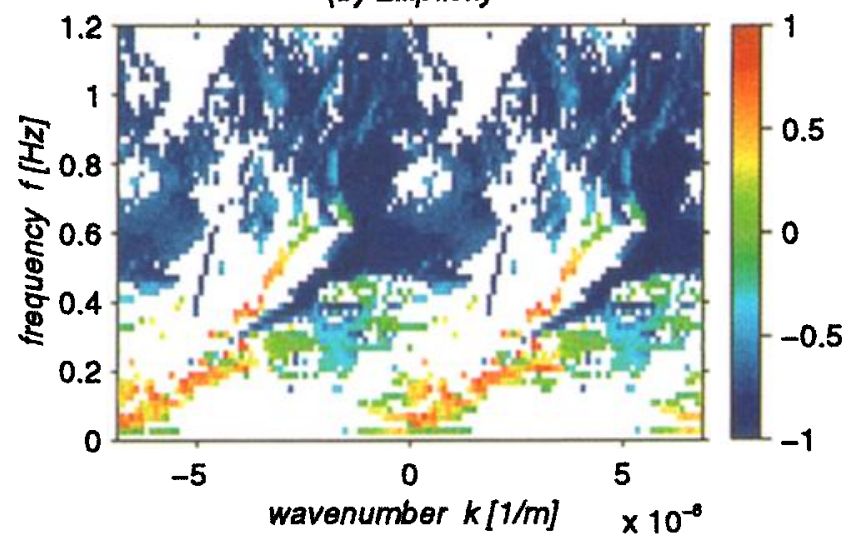

Figure 4. The wavefield polarization (a) and ellipticity (b) associated with Fig. 2. The polarization ranges from 0 for unpolarized to 1 for fully polarized waves. Negative (positive) values of the ellipticity correspond to right- (left-) handed waves in the plasma rest frame. Blank regions denote cells for which the number of samples is too small $(<30)$ or the degree of polarization insufficient $(<0.6)$ to provide reliable estimates. In other regions, the uncertainty on the polarization is at most \pm 0.15 .

\section{Other Spectral Properties}

It was mentioned above that the turbulence is convected earthwards by the solar wind flow. Consequently, the angular frequency $\omega_{0}$ in the plasma rest frame should differ from the one we measure. Both are related by the Doppler shift $\omega=\left|\omega_{0}-\vec{\kappa} \cdot \vec{v}_{S w}\right|$ which can be estimated here since the solar wind velocity $\vec{v}_{S w}$ is known and is almost parallel to $\vec{d}$. The corrected spectrum is displayed in Fig. 3. While the ridges in Fig. $2 \mathrm{a}$ revealed an anti-sunward motion, we now find that the waves actually try to propagate against the solar wind flow, in agreement with previous observations [Schwartz et al., 1992]. The group velocities estimated from Fig. 3 are $v=150-280 \mathrm{~km} / \mathrm{s}$ for the large amplitude fluctuations and $v=80-120 \mathrm{~km} / \mathrm{s}$ for the small amplitude ones. The latter value is in close agreement with the fast magnetosonic speed of the bulk plasma and thus supports the magnetosonic nature of branch $I$.

Although the power spectral density $S(\kappa, \omega)$ provides a wealth of information on the wavefield properties, the picture remains incomplete as long as other quantities such the polarization and the ellipticity are not considered. Their estimation proceeds from that of $S(\kappa, \omega)$ except that now all three components of the 
wavefield are processed. Figure $4 \mathrm{a}$ displays the polarization, which is the fraction of energy stored in fully polarized wave packets over the total energy (e.g. [Arthur et al., 1976]). An outstanding feature is the high polarization observed above $f=0.4$ $\mathrm{Hz}$. Deeper insight is provided by the ellipticity, which ranges between -1 for right-handed circularly polarized waves (in the plasma rest frame) and +1 for left-handed waves, see Figure $4 \mathrm{~b}$. The region which has a high degree of polarization is found to correspond to intrinsic right handed circularly polarized waves. Such properties, together with the dispersion relation allow us to identify these waves as whistlers, which are indeed frequently observed as shocklets in the ultra low frequency waves upstream bow shocks [Hoppe et al., 1983]. As one proceeds downwards in frequency, the ellipticity suddenly changes sign, in agreement with what is predicted for certain classes of soliton-like Alfvén waves [Hada et al., 1989]. These results thus corroborate previous observations and in addition ease their interpretation. A major improvement is the possibility to provide a statistical description for phenomena whose understanding was so far essentially based on a patchwork of individual observations.

\section{Conclusions and Perspectives}

It has been shown how the combination of different existing techniques provides a new and more powerful framework for estimating wavefield parameters in plasma turbulence on the basis of two or more simultaneous measurements. Using this framework, we have established the impact of nonlinear processes on wave propagation and provided the main elements for identifying wavefield properties.

A direct application of this two-point technique to multipoint experiments such as CLUSTER is straightforward and in principle allows the full spectrum $\mathbf{S}(\vec{K}, \omega)$ to be determined. A more rigorous generalization, however, requires a self-consistent and therefore more complex processing of all the different wavefield components. Multivariate approaches [Lefeuvre et al., 1992] may be more appropriate for such purposes. The present work, however, shows that major progress can still be achieved by a suitable combination of techniques such as the wavelet transform, which is particularly appropriate for resolving plasma turbulence.

Acknowledgements. The first author gratefully acknowledges support from the Swiss National Science Foundation. This work was also partly supported by the Department of the Sciences of the Universe (SDU) and the National Institute for Sciences of the Universe (INSU). Finally, we acknowledge discussions with $\mathbf{M}$. Balikhin and J.-L. Pinçon.

\section{References}

Arthur, C. W., R. L. Mc Pherron, and J. D. Means, A comparative study of three techniques for using the spectral matrix in wave analysis, Radio Science 11, 833, 1976.

Beall, J. M., Y. C. Kim, and E. J. Powers, Estimation of wavenumber and frequency spectra using fixed probe pairs, J. Appl. Phys. 53, 3933, 1982.

Carlson, A. W., Two-point estimates of the spectral density function with finite separation and volume, J. Appl. Phys. 70, 4033, 1991.

Dudok de Wit, T., and V. V. Krasnosel'skikh, Wavelet bicoherence analysis of strong plasma turbulence at the Earth's quasi-parallel bow shock, Phys. Plasmas, in press, 1995.

Farge, M., Wavelet transforms and their application to turbulence, Ann. Rev. Fluid Mech. 24, 395, 1992.

Hada, T., C. F. Kennel, and B. Buti, Stationary nonlinear Alfvén waves and solitons, J. Geophys. Res. 94, 65, 1989.

Hoppe, M., and C. T. Russell, Whistler mode wave packets in the Earth's foreshock region, Nature 287, 417, 1980.

Hoppe, M., and C. T. Russell, Plasma rest frame frequencies and polarizations of the low-frequency upstream waves: ISEE 1 and 2 observations, J. Geophys. Res. 88, $2021,1983$.

spectrum for plasma waves and turbulence observed in space plasmas, J. Atm. Terr. Phys. 54, 1227, 1992.

Mann, G., H. Luhr, and W. Baumjohann, Statistical analysis of short large-amplitude magnetic field structures in the vicinity of the quasiparallel bow shock, J. Geophys. Res. 99, 13315, 1994.

Meyer, Y., and S. Roques (Eds.), Progress in wavelet analysis and applications, Editions Frontières, Gif-sur-Yvette, France, 1993.

Mjølhus, E., and J. Wyller, Non-linear Alfvén waves in a finite beta plasma, J. Plasma Phys. 40, 299, 1988.

Omidi, N., and D. Winske, Steepening of kinetic magnetosonic waves into shocklets: simulations and consequences for planetary shocks and comets, J. Geophys. Res. 95, 2281, 1990.

Schwartz, S. J., D. Burgess, W. P. Wilkinson, R. L. Kessel, M. Dunlop, and $H$. Lühr, Observation of short large-amplitude magnetic structures at a quasi-parallel shock, J. Geophys. Res. 97, 4209, 1992.

Sonnerup, B. U. Ö., and L. J. Cahill, Magnetopause structure and attitude from Explorer 12 observations, J. Geophys. Res. 72, 171, 1967.

Thomson, M. F., J. T. Gosling, S. J. Bame, and C. T. Russell, Magnetic pulsations at the quasi-parallel shock, J. Geophys. Res. 95, 957, 1990.

Whitham, G. B., Linear and nonlinear waves, Wiley, New York, 1973.

S. D. Bale and S. J. Schwartz, Astronomy Unit, Queen Mary and Westfield College, Mile End Road, London E1 4NS, UK.

T. Dudok de Wit and V. V. Krasnosel'skikh, LPCE / CNRS, 3A Av. de la Recherche Scientifique, 45071 Orléans, France.

M. W. Dunlop, Space and Atmospheric Physics, Imperial College, Prince Consort Road, London SW7 2BZ, UK.

H. Lühr, Inst. für Geophysik und Meteorologie, Technische Univ. Braunschweig, Mendelssohnstr. 3, 38106 Braunschweig, Germany.

L. J. C. Woolliscroft, Dept. of Automatic Control and Systems Eng., University of Sheffield, Mappin Street, Sheffield S1 3JD, UK.

(Received May 15, 1995; revised June 26, 1995; accepted July 19, 1995.) 